\title{
Transformación de agencias de estudiantes en práctica profesional de Trabajo Social y Psicología: Aportes desde la intervención en violencia de género
}

\author{
Transformation of student agencies into the professional \\ practice of Social Work and Psychology: Contributions from the \\ intervention in gender violence
}

Transformação de agências estudantis em estágio profissional de Serviço Social e Psicologia: Contribuições a partir da intervenção em violência de gênero

Rocío Santander*, Raúl Ulloa**,
Javiera Gatica*** y Andrea Avaria****

\section{RESUMEN}

Se abordan las transformaciones de las agencias de estudiantes Palabras clave: en práctica profesional en intervención en violencia de género, violencia a partir de las repercusiones de la intervención en violencia. Se de género, presentan los resultados de la investigación ${ }^{1}$ de carácter cualiestudiantes, tativo, a través del análisis comparativo constante que permite reflexionar, desde la práctica profesional, en aspectos que impliagencias, intervención. ca revisar el conocimiento, la experiencia y la identificación con la violencia. Se evidencia que la práctica profesional en violencia

\footnotetext{
* Chilena. Trabajadora Social mención Gestión políticas públicas, Universidad Autónoma de Chile. E-mail: rocio.santander.n@gmail.com

** Chileno. Trabajador Social mención Gestión políticas públicas, Universidad Autónoma de Chile.E-mail: raululloats@gmail.com

*** Chilena. Trabajadora Social mención Gestión políticas públicas, Universidad Autónoma de Chile. E-mail: javieraandreagatica@gmail.com

**** Chilena. Dra. en Antropología Social, docente e investigadora, Universidad Autónoma de Chile. E-mail: andreaavaria@gmail.com

${ }^{1}$ Investigación realizada para optar al título profesional de Trabajadores Sociales y al grado académico de licenciado/as en Trabajo Social. La tesis presentada fue aprobada 2019. Los resultados que se presentan responden a parte de esta investigación y no abordan todos los objetivos propuestos en ella.
} 
conlleva la transformación de la manera en que estudiantes en práctica se posicionan, desde la construcción de agencias, en sus historias familiares y desde una estructura social permeada por la violencia.

\begin{abstract}
The transformations of student agencies into professional practice in gender violence intervention are addressed based on violence intervention's repercussions. The qualitative research results are presented through the constant comparative analysis that allows reflecting, from the professional practice, in aspects that imply reviewing the knowledge, the experience, and the identification with the violence. A professional approach to violence entails transforming how students in practice position themselves, from the construction of agencies, their family histories, and social structure is permeated by violence.
\end{abstract}

\section{RESUMO}

As transformações das agências estudantis em estágio profissional em intervenção de violência de gênero são abordadas, com base nas repercussões da intervenção em violência. Os resultados da pesquisa de caráter qualitativo são apresentados através da análise comparativa constante que permite refletir, a partir da prática profissional, sobre aspectos que envolvem a revisão de conhecimentos, experiências e a identificação com a violência. Fica evidente que o estágio profissional em violência implica a transformação da forma como os estagiários se posicionam, desde a construção de agências, em suas histórias familiares e desde uma estrutura social permeada pela violência.
Key words: gender violence, students, agencies, intervention.

Palavras-chave: violência de gênero, estudantes, agências, intervenção. 


\section{Introducción}

Actualmente, la violencia de género y la violencia intrafamiliar se han constituido a nivel global, como una problemática estructural de la sociedad. Se produce una reflexión acerca de quién ha sido o no víctima de algún tipo de violencia, y si quienes han vivido violencia y la han acompañado deben los encargados de la labor de reparación y tratamiento tanto de quienes denuncian o son denunciados. La violencia afecta de forma transversal a toda la sociedad y, en lo particular, también a los profesionales y a quienes se forman en intervención en violencia. Este artículo abordará, desde la conceptualización, la intervención directa en violencia y cómo este ejercicio de carácter profesional repercute en la vida de estudiantes en proceso de formación. Este proceso evidencia que los procesos asociados a la intervención, como veremos a lo largo del texto, permiten la construcción de agencia y, con ello, la modificación de su posición en la estructura social relacionada al ejercicio de la violencia.

En Chile, en 1991 se crea el Servicio Nacional de la Mujer, actualmente Servicio Nacional de la Mujer y la Equidad de Género (SernamEG), se constituye así un organismo que, como parte del Ministerio de la Mujer y Equidad de Género, permite dar respuesta a esta problemática. El Estado chileno incorpora, con ello, un nuevo marco jurídico y de intervención a través de diferentes dispositivos territoriales que apuntan a la promoción, prevención y protección de los derechos de las personas que habitan el territorio nacional.

Un mecanismo que permite aproximarse a las personas y atender los efectos de la violencia de género son los centros de atención de mujeres que viven violencia. La oferta programática se constituye a través de la implementación de centros de atención reparatoria a mujeres víctimas de agresiones sexuales (CVS), centros de la mujer (CDM), casas de acogidas, entre otras. A su vez, existen centros de atención exclusiva a hombres que hayan ejercido violencia en contra de sus parejas, centros de reeducación de hombres que ejercen violencia de pareja (HEVPA), cuyo principal objetivo es contribuir a la total eliminación de todos los tipos de violencia, a través de estrategias para minimizar los niveles de violencia ejercidos contra de las mujeres, favorecer así la seguridad y protección de estas (SernamEG, s.f.). Estas intervenciones consideran como esencial el reconocimiento de la violencia ejercida. 
En este marco de apoyo al incremento en el acceso a atención en atención en violencia, el Centro de Prevención y Tratamiento de Violencia Intrafamiliar (que nos ocupa en la investigación) pertenece a una universidad privada y se encuentra bajo la directriz de la Facultad de Ciencias Sociales y Humanidades, a través de las carreras de Trabajo Social y Psicología, creado de forma privada para dar respuesta a las necesidades existentes ${ }^{6}$, ofrece atención psicosocial tanto a hombres como a mujeres que viven o ejercen violencia en contextos de pareja, tanto en su calidad de víctimas o, bien, debido a que ejercen violencia y asisten en su calidad de denunciados o imputados. Este Centro, a través de prácticas profesionales de estudiantes de pregrado, tanto de Trabajo Social como de Psicología, es un espacio de formación intermedia y final, supervisado por una dupla psicosocial que, a su vez, realiza la coordinación del centro.

El equipo psicosocial en formación se compone por siete estudiantes de Trabajo Social y siete de Psicología, siendo jóvenes de entre 21 a 30 años, en su mayoría de familias de tipo monoparental nuclear que residen dentro de la Región Metropolitana, principalmente en la comuna de Puente Alto (11,7\%), San Bernardo (7,3\%), Maipú (6,7\%), entre otras. Además, señalan tener experiencias personales en relación con la violencia, las cuales están relacionadas con el interés y motivación por la temática y con aportar en la resolución de problemáticas en violencia intrafamiliar.

\section{Intervención en violencia de género/violencia intrafamiliar}

Las intervenciones en violencia hacia las mujeres son realizadas por lo general por las disciplinas de Trabajo Social y Psicología. La intervención psicosocial permite desplegar y complementar las aproximaciones sociales y psicológicas que se entrecruzan en los procesos de violencia (Peláez, Cañon, y Noreña, 2007). La oportunidad de desplegar una aproximación psicosocial permite recoger en el proceso de intervención tanto las creencias, significados a partir de las experiencias y

6 Requerimientos del Primer Tribunal de Garantía en cuanto a la atención especializada a hombres imputados por violencia intrafamiliar y a la elaboración de informes periciales. 
vivencias de las mujeres y hombres que acuden a atención psicosocial, permitiendo ampliar y entrecruzar las complejidades sociales, contextuales, culturales, y también aquellas de carácter más individual y psicológicas de los usuarios y usuarias.

La intervención en violencia hacia las mujeres tiene un sustento en el enfoque de género, el cual se vuelve imprescindible para el entendimiento de los casos en un marco más global, contextual, y estructural de relaciones entre mujeres y hombres. Este enfoque permite comprender que las problemáticas sociales en el marco de la violencia se relacionan con los múltiples aspectos que las mujeres enfrentan, tales como dificultades y limitantes en el acceso a recursos económicos, sociales y educativos. El género, como perspectiva de interpretación de las condiciones de violencia que afectan a las mujeres, permite ampliar la comprensión y la interpretación de las condiciones que las afectan, y no culpabilizar a las mujeres en su condición de víctimas (Burin, en Cañas, 2010).

Los contextos de violencia requieren de miradas complejas que permitan desarrollar intervenciones que incorporen esta complejidad. Abordar la complejidad desde una perspectiva interdisciplinaria, amplía y potencia este abordaje. La interdisciplinaridad permite ampliar, transformar y complementar diversos enfoques de intervención, con el fin de priorizar la atención conjunta e integral (Jiménez, Pallares y Bustamante, 2005); permite la interacción de saberes propios de cada ámbito profesional. La interacción e influencia recíproca promueven la incorporación de marcos epistemológicos.

Por su parte, el modelo ecológico ${ }^{7}$ permite dar cuenta de los factores que se asocian a la aparición y persistencia de la violencia en los ámbitos individual, familiar, biológico, cultural, socioeconómico y político; y, conjuntamente, permite diseñar estrategias de intervención y prevención. Asimismo, afirma que el desarrollo humano se produce mediante la interacción de diversos sistemas y niveles ecológicos, a los cuales Bronfernbrenner (1977) denominó: microsistema, mesosistema, exosistema y macrosistemas, respectivamente. El modelo ecológico permite analizar la violencia intrafamiliar, al establecer una relación

7 Modelo desarrollado por Urie Bronfenbrenner, en el año 1977, en su obra 'La ecología del desarrollo humano". 
entre los factores ambientales con los que pudiese o no interactuar la persona que experimenta o ejerce violencia, de este modo, releva la necesidad de indagar la influencia de estos factores, ya que identificarlos favorece distinguir los procesos adaptativos y desadaptativos, y facilita reconocer las redes de apoyo primarias, secundarias e institucionales con las que se vinculan o no las personas.

\section{Intervenir en violencia desde las agencias}

La intervención tiene como propósito la transformación. Ello conlleva involucrar en el proceso tanto a las personas sujetos de atención, como también considerar, en este proceso, la relación entre profesionales (ello incluye al o la practicante) y las personas usuarias. La relación que ocurre entre estos sujetos modifica a ambos en esa interacción.

La práctica social es entendida por Zavala (2011) como un aprendizaje en espacios que posibilitan el desarrollo de habilidades e identidades, y que favorecen la interacción con comunidades específicas en un particular marco de valores. Para estos efectos, consideraremos la práctica social en relación con la intervención en violencia de género y/o intrafamiliar.

Por otra parte, entenderemos como "agencia" aquella capacidad de las personas, grupos o instituciones para potenciar sus metas y objetivos. La agencia se conforma por redes de instituciones y actores que, a través de sus acciones e interacciones, producen desarrollo. Especial énfasis tienen las capacidades relativas a los procesos de desarrollo autoconstruido, y cobran especial atención aquellas que se relacionan con sus principales protagonistas, en un marco de diálogo y de negociación permanente (Bivort, 2005).

La agencia de las personas se relaciona con la personalidad, la voluntad, la libre determinación y la naturaleza de su conciencia (Fowler, 2013). Estas acciones, según Zavala (2011), consideran también las transgresiones que las personas producen en el marco de las estructuras sociales en que se encuentran.

En el marco de este estudio, la práctica profesional, en un contexto de atención de las personas afectadas por la violencia como fenómeno social, conlleva la necesidad de identificar los aspectos asociados a la 
agencia de estos estudiantes, especialmente identificar aquellas acciones que se relacionan, como actos reflexivos y de cuestionamiento, en el marco del lugar que ocupan en las estructuras sociales relativas a la violencia.

\section{¿Qué sucede en quien interviene en violencia?}

En el marco del ejercicio profesional y de práctica profesional de quienes se forman en Trabajo social y Psicología, y en la interacción que se produce en la intervención, es posible subrayar procesos de identificación con las personas a quienes se trata o con quienes se interviene. En la interacción, en las historias personales, se observa que la violencia ha afectado tanto a quien ha vivido o ejercido violencia, o forma parte de la vida de quienes intervienen y de quienes son usuarios de un determinado servicio. Este proceso de identificación implica observar-observarse y relacionarse desde un lugar más próximo; con ello se rompe la distinción entre el otro-víctima/victimario y el nosotros (neutro).

Tanto los profesionales como los practicantes, al estar en contacto con su propio sufrimiento (en el contexto de atención de otros), y con el de las personas usuarias, a largo plazo, como consecuencia, podrán desarrollar el síndrome de Burnout. Este afecta a profesionales que intervienen en temáticas complejas, manifestándose principalmente en agotamiento físico y psicológico, una actitud lejana y despersonalizada durante la interacción, o en sus relaciones interpersonales; conlleva un estado de inadecuación en el ejercicio profesional, como consecuencia del estrés emocional crónico (Maslach y Jackson, en Morales, Pérez, Menares, 2003), lo que provoca sentimientos de desesperanza y apatía, al no alcanzar, los profesionales o practicantes, los propósitos más allá del esfuerzo realizado para ello (Filgreira, 2014).

La respuesta de desgaste al escuchar episodios traumáticos vivenciados por otra persona se conoce como el "desgaste de empatía o estrés traumático secundario (ETS) (Figley, en Arita, Casillas y Romero, s.f.). En los procesos de intervención en violencia hacia las mujeres, en que se escucha tanto a quien sufrió o sufre violencia, como también a quien ejerció o ejerce violencia, estos procesos de intervención se transforman en espacios en los que desembocan emociones, padecimientos. En ellos se abandonan los límites emocionales, se rompen los 
límites entre la persona usuaria y quien interviene, desarrollándose así procesos de desgaste y, por ende, estrés traumático secundario (Arita, Casillas y Romero, s.f.).

El estrés traumático secundario se produce por factores externos: las jornadas laborales extenuantes, traumáticas y estresantes; en ello influye también la despreocupación por el autocuidado de los profesionales. En los procesos de intervención se despliegan factores internos, relacionados con la historia de vida, la personalidad de los sujetos, los episodios traumáticos sin elaborar y los mecanismos de afrontamiento relativos a la contaminación, que afecta y vincula la temática que aborda con la propia historia (Castillas, 2016).

Quienes intervienen en violencia intrafamiliar también podrían sufrir desgaste emocional o estados de extenuación emocional, entendidos como falta de energía y falta de satisfacción por el trabajo: se desencadena fatiga y dilatación frente a tareas relacionadas con el quehacer (Wilson en Claramunt, 1999). Este desgaste también se puede evidenciar en la despersonalización y el distanciamiento emocional con los relatos de los usuarios, distancia con la temática en la que interviene el profesional, así como también, una distancia con el equipo con el cual se desempeña. Como consecuencia de intervenir en violencia hacia las mujeres se produce, en quien interviene, una sensación de pérdida de control, puesto que el profesional se reconoce en la indefensión de la persona usuaria, o, por el contrario, a través de la intervención se percata de su situación presente o futura, de alguna manera se ve influenciada por las acciones de otros y experimenta de igual forma la falta de control (Vexina, 2014).

Estas cuestiones son especialmente sensibles y relevantes cuando observamos a las duplas psicosociales en formación. Para quienes son profesionales en formación y que como estudiantes aún no cuentan con experiencia acumulada o con herramientas especificas, o aún no reciben formación especializada en la temática, intervenir en violencia se refleja y relaciona con las propias historias de vida. Para quienes desarrollan prácticas profesionales en violencia se produce una identificación con la problemática de las personas usuarias, a través de ellas se reflejan las emociones, sentimientos, padecimientos. Se produce una contraposición entre las experiencias y necesidades propias, y en el quehacer profesional (práctica profesional), y estas se contraponen 
con los requerimientos de las personas que atienden e incluso se tensionan en el marco ético profesional (Daskal, 2017).

\section{Antecedentes}

El estudiantado en práctica profesional, si bien realiza las mismas intervenciones en violencia que los profesionales de Trabajo Social y Psicología, no suelen ser considerados como parte de quienes sufren las consecuencias de intervenir. Los estudiantes experimentan diversos estados. $\mathrm{Al}$ aproximarse a la realidad de los usuarios, presentan angustia, dudas y desesperación frente a la problemática en la que intervienen; a ello se suma la poca experiencia práctica en el ejercicio de su profesión(Burgos, 2013). Todo esto tiene consecuencias que pueden ser positivas o negativas. Como consecuencias positivas es posible afirmar que el estudiante en práctica puede, mejorar y/o profundizar la forma en que se relaciona en su contexto familiar, pues podrá desarrollar mayor sensibilidad y paciencia. En esta línea también, frente al recuerdo y comparación entre las experiencias familiares y la propia historia familiar, podrá incrementar sus capacidades y disposición a solucionar conflictos familiares históricos (Burgos, 2013). Al estar en un estado de alerta durante sesiones, y ante las similitudes entre las dificultades de su familia de origen y los usuarios, se aprenden otras formas de vincularse, de forma asertiva y sin la utilización de la violencia, como también, se identifican las situaciones de violencia en el marco de las actuales relaciones, modificando los mecanismos de interacción y relación entre las personas.

Respecto de las consecuencias negativas, el estudiantado en formación podría enfrentarse a

sentimientos de superioridad, presión interna en caso de haber familiares con problemas (...) lo que puede ser causal de una desestabilización en las relaciones, debido a que su conducta puede ser interpretada como un ataque a miembros específicos de la familia, o puede que existan miembros que no desean abordar la temática que éste plantea, entre otras situaciones que terminarían profundizando las diferencias o produciendo nuevos enfrentamientos entre los miembros del clan familiar. (p. 29)

En este contexto, los estudiantes podrían enfrentarse a situaciones de presión de algún miembro de sus familias, especialmente de 
quienes enfrentan problemas de violencia. Al posicionarse desde este lugar, es posible que generen sentimientos de superioridad, lo que podría ser interpretado como una conducta ofensiva al interior de su grupo familiar, pudiendo ocasionar crisis dentro de la misma; es decir, no todos quienes forman sus familias estarán dispuestos a afrontar la problemática que los aqueja, ni a ser interpelados, lo que conlleva una serie de consecuencias complejas.

El agente en la estructura social y familiar es el profesional en formación, quien no es ajeno a intentar modificar su propia realidad: "los actores no son ni agentes totalmente libres ni productos sociales completamente determinados por la estructura; en algunas circunstancias pueden transformar los sistemas que los producen"(Zavala, 2011, p. 56), lo cual muestra la posibilidad de generar cambios en la sociedad, y específicamente en las relaciones dentro de su círculo familiar. Por ello, quién adquiere conocimientos relativos a la problemática de la violencia de género puede volverse una persona más dispuesta a percibir situaciones de violencia y, a partir de ello, querer tomar acciones frente la problemática. De este modo, la persona se constituye en agencia dentro de lo social. Fowler (2013) refiere que esta es la "capacidad de actuar basada en el control de los recursos estratégicos" (p. 119), por lo que el individuo posee la facultad de decidir libremente, en su propio contexto, y este responde a características que se dan al interior de su historia de vida.

La historia de vida de quienes intervienen se constituye por un lado como una construcción histórica de sí mismo, en la que se producen interpretaciones y significados de hechos, situaciones y vivencias ocurridos en los diversos ámbitos de la vida cotidiana. Esta historia se relaciona con los marcos histórico, cultural, socioeconómico y demográfico que condicionan su comportamiento, en las diversas áreas en las que se desenvuelven. Los acontecimientos y/o experiencias relacionadas con la violencia al interior de sus familias pueden estar relacionados con episodios aislados de agresiones y/o maltrato, a pesar de que, al interior de estas, se espera hallar principalmente amor, confianza y seguridad. Por lo que episodios no resueltos de violencia limitan la capacidad de ayuda en una persona distinta; quienes intervienen con usuarios que sufren o ejercen violencia podrían conducir su dolor hacia estos, e intentar resolver sus problemas a través de esa conducción (Claramunt, 1999). 
El reconocimiento de una persona distinta, como otro, se produce en el ejercicio de una escucha activa de su historia de vida, de lo que "proviene de lo visto y lo oído, incluyendo aquello que se reconoce como la vida íntima, y esto lleva a una incierta transformación que se considera apropiada para la aparición pública" (Arendt, en Cárdenas, 2018, p. 49). Resignificar contribuye entonces a una deconstrucción de lo ya construido, determinado o aceptado en la historia de vida,

también en la medida que el terapeuta avanza en el ciclo vital, se puede ver enfrentado a problemas o preocupaciones que pueden desorganizar la estabilidad tanto de sus vidas como de sus relaciones, por ende, significar una fuente considerable de tensión y entorpecer tanto su vida personal como profesional. (Guy, en Carvajal, 2012, p. 20)

Como hemos afirmado y en el marco de lo planteado por Vexina (2014), quienes realizan procesos interventivos o terapéuticos, además de estar en contacto con el sufrimiento de otras personas, lo están con su propio sufrimiento; deben afrontar no solo tensiones o ansiedades propias, por su quehacer, sino también sus sentimientos y emociones que se generan al trabajar con el padecimiento de los seres humanos.

Zurh (1994) plantea al menos cuatro repercusiones o ramificaciones de intervenir en violencia, en psicoterapeutas y sus familias: la primera es la interpretación, que se relaciona con el ejercicio, de parte de terapeutas, de interpretar los sueños o situaciones que involucran responsabilidad en sus familiares, analizando profundamente estas situaciones sin permiso de los protagonistas; ello implica tratar a los familiares como si fueran personas usuarias. La segunda es el cuestionamiento y consulta, la cual se refiere a la preparación teórica para realizar interrogantes con el fin de profundizar en ciertos tópicos. Se plantean complejas preguntas para guiar la reflexión de sus familiares. La tercera repercusión negativa, es el drenaje emocional, que consiste en que el profesional reprime sus sentimientos y conversaciones y, con ello, minimiza sus propios problemas familiares, pues los compara con los de las personas a quienes atiende. La cuarta consecuencia es el distanciamiento, que consiste en que el profesional se distancia emocionalmente de su realidad y de su vida familiar. El entendimiento total y no crítico, en tanto repercusión, se produce en la persona del terapeuta que mantiene una distancia analítica con los miembros de su familia, 
bajo la convicción de comprender la situación, ya que la conoce teóricamente y por medio de la praxis.

Los profesionales en formación no logran necesariamente separarse de sus sentimientos, percepciones, pensamientos y de su historia de vida; continuamente revelan su historia. Se elabora una relación transformadora de la realidad, tanto para los usuarios y usuarias, como para los profesionales en práctica (Aron, 1996, en Szmulewicz, 2013). Se producen diversas significaciones en el estudiantado, tanto personal como familiares, desde un contexto histórico y sociocultural, las cuales podrán manifestarse de manera consciente o inconsciente, puesto que, al intervenir, se producen consecuencias en la vida del terapeuta, en el ejercicio profesional, puesto que se establece un vínculo entre la vida personal y la de los usuarios (White, 2002, en Szmulewicz, 2013).

Canevaro, Abeliuk, Condeza, Escala, García y Montes (2016) realzan la importancia de la familia de origen de los estudiantes de terapia familiar. La familia de origen de los estudiantes deja huella en la personalidad: influye tanto en la vida privada como profesional de las personas y, a su vez, determina que quienes realizan terapia familiar, tienen "una fantasía salvadora de querer resolver los problemas relacionales en su familia de origen" (p. 141).

Por consiguiente, la elección de intervenir en la temática de violencia, los perfiles de las personas usuarias que atienden y los tópicos a tratar en cada sesión no son decisiones azarosas, aunque se rigen bajo los parámetros de intervención de la institución a la que adscriben, ya que estas responden a la identificación de su problemática con la de los usuarios y provocan un cuestionamiento en relación con los patrones relaciones, los roles y los conflictos familiares.

Por su parte, Burgos (2013) compara los efectos de la problemática en los usuarios, en psicólogos en formación y en profesionales. El autor determinó que el estudiantado, luego de realizar intervenciones, modificó sus creencias políticas, religiosas, sociales y personales, lo que da cuenta de la significativa influencia de la persona atendida en la vida personal de quien interviene.

Los terapeutas plantean que, al encontrarse con pacientes que describen problemáticas similares a las propias o que los afectan emocionalmente en mayor medida, la sesión les resulta más des- 
gastante y agotadora, debido a que deben realizar la doble tarea de poner atención al paciente y a lo que les ocurre internamente. Por otro lado, la afección familiar en el grupo de novatos surgió en uno de los participantes, quien indicó que debía reprimir los temas que experimentaba en su rol de terapeuta, ya que percibía que su trabajo no era comprendido en su contexto familiar. (Burgos, 2013, p. 76)

Se evidencia la necesidad de enfatizar en las duplas psicosociales en formación. Es relevante identificar la circularidad de la violencia, tanto de quien ejerce, del que sufre producto de la violencia y de quien interviene, al existir un ejercicio personal sumado a los procesos de intervención, dado que la violencia trasciende todas las estructuras sociales.

La evidencia indica que las personas que intervienen en temáticas complejas presentan a lo menos una de las consecuencias de síndrome de Burnout, se presentan sentimientos como tristeza, angustia, agotamiento emocional, físico y psicológico. Como también los síntomas asociados a estrés traumático secundario y/o estado de extenuación emocional.

Esta reflexión nos sitúa en la necesidad de analizar la forma en que se modifican las agencias de los estudiantes del campo de la Psicología y el Trabajo social, luego de realizar intervenciones en violencia de género en el Centro de Prevención y Tratamiento de la Violencia Intrafamiliar.

\section{Metodología}

La investigación se abordó desde el enfoque cualitativo-interpretativo, el que parte de la base de que el mundo social se construye de significados y símbolos, lo que conlleva a la búsqueda de esta construcción de significados (Ruiz, 2012).

Desde el diseño fenomenológico se permite una visión desde los participantes, y esta permite comprender, entender y describir los fenómenos sociales. Consiste en dos visiones: la fenomenología hermenéutica, en la cual la interpretación se centra en la experiencia, y la fenomenología empírica, que se enfoca en la descripción de las vivencias de quienes participan (Hernández, Fernández y Baptista, 20014).

El estudio se ha propuesto analizar las repercusiones asociadas a intervenir en violencia de género, en estudiantes de Trabajo Social y 
Psicología que realizaron su práctica profesional en el Centro de Prevención y Tratamiento de la Violencia Intrafamiliar. Para esto se consideró avanzar en la comprensión de tres ejes. El primero consistió en identificar las modificaciones de las agencias de los estudiantes luego de realizar intervenciones; un segundo eje consistió en explicar el lugar que ocupa la violencia en las relaciones interpersonales de los estudiantes luego de realizar intervenciones, y el tercero se propuso establecer las transformaciones en las construcciones de género de los practicantes. En el presente artículo se abordará, de forma parcial, el contenido el primer eje.

Las técnicas de recolección, de carácter cualitativo, fueron principalmente la entrevista en profundidad y la entrevista semiestructurada. El instrumento utilizado fue puesto a prueba durante agosto del 2019, con practicantes de años anteriores, pertenecientes a ambas disciplinas; este fue ajustado y aprobado para su aplicación.

El trabajo de campo se realizó durante el mes de septiembre del mismo año. Se entrevistó a siete estudiantes de Trabajo Social y siete de Psicología, quienes realizaron su práctica profesional en el Centro de Prevención y Tratamiento de la Violencia Intrafamiliar. Para enriquecer el análisis y la recolección de información, se entrevistó a la coordinadora de Trabajo Social del mismo Centro, en su calidad de informante clave.

La información recopilada, fue analizada mediante la teoría social fundamentada, ya que implica un método cuyo fin es originar, de manera sistemática, las teorías sobre el comportamiento humano y el mundo social con una base empírica (Kendall, 1999, en Torre et al., 2011). Al existir un vacío teórico respecto de la temática abordada, la relevancia de la teoría se presenta en la "vinculación entre un sujeto que busca la comprensión de un objeto a investigar mediante las acciones y significaciones de los participantes de la investigación" (Charmaz, en Bonilla y López, 2016, p. 306). Se realizó un análisis sistémico de la información a través del método comparativo constante, en el que "el investigador, simultáneamente codifica y analiza datos para desarrollar conceptos. Mediante la comparación continua de incidentes específicos de los datos, el investigador refina esos conceptos, identifica sus propiedades, explora sus interrelaciones y los integra en una teoría coherente" (Cuñat, 2007, p. 2). 
La codificación axial genera un análisis de los datos obtenidos para responder a los objetivos de investigación planteados. En este marco, presentamos un esquema que permite destacar las diferentes categorías y su interrelación, cada esquema se muestra de forma diferenciada, de acuerdo con la experiencia del estudiantado de cada disciplina de estudio:

Tabla 1

Codificación axial estudiantes de Trabajo Social

\begin{tabular}{|c|c|c|c|}
\hline Categorías iniciales & $\begin{array}{l}\text { Subcategorías } \\
\text { iniciales }\end{array}$ & $\begin{array}{l}\text { Categorías } \\
\text { emergentes }\end{array}$ & $\begin{array}{l}\text { Subcategorías } \\
\text { emergentes }\end{array}$ \\
\hline $\begin{array}{l}\text { Redefinición } \\
\text { de relaciones } \\
\text { interpersonales }\end{array}$ & \multirow{3}{*}{$\begin{array}{l}\text { Aprendizaje } \\
\text { personal }\end{array}$} & \multirow{3}{*}{$\begin{array}{l}\text { Dinámica } \\
\text { familiar }\end{array}$} & $\begin{array}{l}\text { Cuestionamiento } \\
\text { en la comunicación } \\
\text { familiar }\end{array}$ \\
\hline Roles y estereotipo & & & $\begin{array}{l}\text { Concientización } \\
\text { de episodios de } \\
\text { violencia en su } \\
\text { familia }\end{array}$ \\
\hline $\begin{array}{l}\text { Intervención en su } \\
\text { familia }\end{array}$ & & & $\begin{array}{l}\text { Proceso de } \\
\text { crecimiento/ } \\
\text { sentimiento de } \\
\text { responsabilidad }\end{array}$ \\
\hline $\begin{array}{l}\text { Rol del trabajador/a } \\
\text { social }\end{array}$ & \multirow{4}{*}{$\begin{array}{l}\text { Aprendizaje } \\
\text { profesional }\end{array}$} & \multirow{7}{*}{$\begin{array}{l}\text { Experiencias } \\
\text { personales } \\
\text { de violencia } \\
\text { intrafamiliar }\end{array}$} & $\begin{array}{l}\text { Identificación con } \\
\text { los usuarios/as }\end{array}$ \\
\hline $\begin{array}{l}\text { Rol de dupla } \\
\text { (psicólogos/as) }\end{array}$ & & & $\begin{array}{l}\text { Emocionalidad } \\
\text { /miedo /rabia }\end{array}$ \\
\hline $\begin{array}{l}\text { Trabajo } \\
\text { interdisciplinario }\end{array}$ & & & $\begin{array}{l}\text { Agotamiento y } \\
\text { desesperanza }\end{array}$ \\
\hline Intervención directa & & & \multirow{4}{*}{$\begin{array}{l}\text { Motivación e } \\
\text { interés }\end{array}$} \\
\hline Violencia estructural & \multirow{3}{*}{$\begin{array}{l}\text { Aprendizaje } \\
\text { en violencia }\end{array}$} & & \\
\hline $\begin{array}{l}\text { Consecuencias de la } \\
\text { violencia }\end{array}$ & & & \\
\hline $\begin{array}{l}\text { Complejidad de la } \\
\text { temática }\end{array}$ & & & \\
\hline
\end{tabular}

Fuente: Elaboración propia. 
Figura 2:

Codificación axial estudiantes de Psicología

\begin{tabular}{|c|c|c|c|}
\hline Categorías iniciales & $\begin{array}{l}\text { Subcategorías } \\
\text { iniciales }\end{array}$ & $\begin{array}{l}\text { Categorías } \\
\text { emergentes }\end{array}$ & $\begin{array}{l}\text { Subcategorías } \\
\text { emergentes }\end{array}$ \\
\hline $\begin{array}{l}\text { Redefinición } \\
\text { de relaciones } \\
\text { interpersonales }\end{array}$ & \multirow{4}{*}{$\begin{array}{l}\text { Aprendizaje } \\
\text { personal }\end{array}$} & \multirow{4}{*}{$\begin{array}{l}\text { Historia } \\
\text { familiar }\end{array}$} & $\begin{array}{l}\text { Cuestionamientos } \\
\text { a comportamientos } \\
\text { violentos en sus } \\
\text { familias }\end{array}$ \\
\hline $\begin{array}{l}\text { Intervención sobre } \\
\text { violencia en su } \\
\text { familia }\end{array}$ & & & $\begin{array}{l}\text { Cuestionamientos } \\
\text { al uso de la } \\
\text { violencia }\end{array}$ \\
\hline $\begin{array}{l}\text { Mecanismos de } \\
\text { protección }\end{array}$ & & & \multirow{2}{*}{$\begin{array}{l}\text { Madurez y } \\
\text { crecimiento } \\
\text { personal }\end{array}$} \\
\hline Roles y estereotipos & & & \\
\hline Rol de psicólogo/a & \multirow{4}{*}{$\begin{array}{l}\text { Prácticas } \\
\text { profesionales }\end{array}$} & \multirow{7}{*}{$\begin{array}{l}\text { Intervenir } \\
\text { en violencia } \\
\text { intrafamiliar }\end{array}$} & $\begin{array}{l}\text { Identificación con } \\
\text { los usuarios/as }\end{array}$ \\
\hline $\begin{array}{l}\text { Límites con los } \\
\text { usuarios/as }\end{array}$ & & & Interés/motivación \\
\hline $\begin{array}{l}\text { Trabajo } \\
\text { interdisciplinario }\end{array}$ & & & $\begin{array}{l}\text { Miedo/ } \\
\text { preocupación }\end{array}$ \\
\hline $\begin{array}{l}\text { Diagnóstico } \\
\text { (evaluación) }\end{array}$ & & & Colapso \\
\hline $\begin{array}{l}\text { Visión estereotipada } \\
\text { de agresor/a y } \\
\text { víctima }\end{array}$ & \multirow{3}{*}{$\begin{array}{l}\text { Aprendizaje } \\
\text { en violencia }\end{array}$} & & Desesperanza \\
\hline $\begin{array}{l}\text { Nuevas formas de } \\
\text { violencia }\end{array}$ & & & \multirow[t]{2}{*}{ Inseguridad } \\
\hline Violencia estructural & & & \\
\hline
\end{tabular}

Fuente: Elaboración propia.

La codificación selectiva se realizó a la luz del marco teórico, considerando la estructura social ${ }^{8}$, teoría de género ${ }^{9}$, teoría de la sociedad del cansancio ${ }^{10}$, y se relacionó con cada uno de los ejes analíticos: mo-

8 Pierre Bourdieu, El sentido práctico (1980), Razones prácticas sobre la teoría de la acción (1994).

9 Marcela Lagarde, Cuadernos inacabados, género y feminismos: desarrollo humano y democracia (1996), Marta Lamas, Diferencias de sexo género y diferencia sexual.

10 Planteada por Byung-Chul Han, en 2012. La sociedad actual es una "sociedad de rendimiento" (p.16) en donde las enfermedades emblemáticas ya no son bacterianas ni virales, sino neuronales, la depresión, el síndrome del desgaste ocupacional o Burnout, entre otros, las cuales tienen la particularidad de que "no son infecciones, son infartos 
dificación de las agencias, violencia en relaciones interpersonales, y la resignificación de las construcciones de género. A partir de lo cual se realizó, posteriormente, la creación de una teoría sustantiva o construcción de afirmaciones.

Se utilizó la triangulación como estrategia que permite una mayor validez del estudio. Para ello se visualizó el fenómeno desde diferentes ángulos, se interpretó y profundizó su comprensión por medio de la triangulación de datos, es decir, el contraste de la información recabada entre los estudiantes de Trabajo Social y Psicología, y a su vez se cotejó esta información con el acercamiento a informantes claves. Se accedió solamente a la coordinadora del Centro de Prevención y Tratamiento de la Violencia Intrafamiliar. Por otro lado, y de forma de complementaria a esto, se realizó la triangulación de investigadores; esta tuvo por fin proporcionar una mayor riqueza analítica e interpretativa, permitiendo así fortalecer el valor y exactitud de los datos recabados (Hernández et al., 2014).

En relación con los resguardos éticos, se construyó y aplicó un consentimiento informado para los estudiantes y las informantes clave. Este documento consideró las implicancias de participar en la investigación, el resguardo de la confidencialidad, identidad e integridad, enfatizando en la voluntariedad de dicha participación especialmente, pues la muestra es parte del estudiantado y docentes que forman parte de la institución, por lo que se requiere especial consideración de modo de salvaguardar las identidades y derechos de las personas entrevistadas. Estos fueron firmados en tres copias, el respaldo, tanto de las entrevistas como de los consentimientos, fue guardado en un lugar de acceso limitado y será destruido en 2025, considerando que en este periodo podrá ser utilizada la información para uso de carácter académico.

\section{Principales resultados}

Los resultados que a continuación destacamos constituyen la teorización del trabajo de campo a partir de las entrevistas realizadas con los estudiantes en práctica profesional en el Centro de Prevención y Tra-

ocasionados no por la negatividad de lo otro inmunológico, sino por el exceso de positividad" (p. 7). 
tamiento de la Violencia Intrafamiliar. Inicialmente, el análisis de la información a partir de la interpretación de la codificación axial, cuya finalidad ha sido ahondar en las repercusiones asociadas a intervenir en violencia de género. Para efectos de este artículo, se presentan de forma parcial. Dada su extensión, se ha decidido sintetizar los resultados de los ejes asociados principalmente a los referidos a la modificación de las agencias desde una perspectiva de género, que, junto con los conocimientos adquiridos, las experiencias personales en el Centro de Prevención y Tratamiento de la Violencia Intrafamiliar y la identificación con los usuarios, se relacionan con la modificación de las agencias de los estudiantes dentro de las familias, sobre la base de sus propias prácticas en el fenómeno de violencia de género, y posteriormente a la realización de intervenciones profesionales como parte de la formación profesional.

Como repercusión asociada a intervenir en violencia de género es la agencia, puesto que, luego de este ejercicio preprofesional, el estudiantado decide tener una acción activa al interior de sus familias, marcando con ello una diferencia entre la realidad que vivían de forma previa a la realización de la práctica profesional. De esta forma, resignifican sus vivencias y, con ello, hay una decisión de no perpetuar la violencia vivida al interior de sus familias. La agencia se produce al confluir los conocimientos adquiridos sobre violencia intrafamiliar y de género, las experiencias en violencia al interior de sus familias o violencia de género, y la identificación con los usuarios en el marco de desarrollo de sus prácticas profesionales.

A continuación presentamos los resultados analíticos, estos serán abordados sintéticamente y en orden diferenciado, de acuerdo con las experiencias diferenciadas entre el estudiantado de Trabajo social y de Psicología, respectivamente.

\section{Estudiantes de Trabajo Social}

A partir de las experiencias recogidas por quienes estudian Trabajo Social, fue posible evidenciar que éstos, al contar con herramientas de reflexión más críticas en relación con las estructuras sociales y el entorno, producto de su formación disciplinar, y dado que en los procesos de formación profesional se producen una serie de prácticas, de acuerdo con el plan formativo al interior de la universidad, estas prácticas previas a la práctica profesional de cierre permiten habilitar e 
integrar, de forma procesual, herramientas que les han habilitado en la intervención social y, también, les han permitido incorporar una actitud más activa y crítica en relación con sus propios entornos familiares.

Parte de estos aspectos serán abordados a continuación, nos referiremos a los conocimientos adquiridos, las experiencias y la identificación del estudiantado con las personas usuarias.

\section{Conocimientos adquiridos sobre violencia intrafamiliar y de género}

Los conocimientos adquiridos durante el proceso de formación permiten a los estudiantes identificar la violencia y adquirir herramientas para intervenir, lo que amplía sus repertorios de respuesta frente a la violencia, especialmente en los procesos de práctica profesional. Estos aprendizajes también transforman la manera en que interactúan dentro de sus familias.

Respecto de la relación con la violencia de género y la concepción que tenían de ésta, primeramente los practicantes de Trabajo Social la refieren como:

La violencia últimamente es todo, o sea violencia desde que la mujer tenga que realizar labores en la casa no remuneradas, es violento estar en un sistema patriarcal donde tenemos roles de género, es muy violento. Más allá del ejercicio de la violencia, es como ejercer la ... como desde el poder, la violencia. Violento es todo, o sea, yo puedo ser violenta con mi amiga (...), somos una sociedad violenta, pero sí, yo creo que las personas violentas se hacen, es como con el medio [influye], no es que una persona nace violenta. No. Sino que es la sociedad que es violenta. (TS-2M)

La entrevistada se refiere a la violencia como una desigualdad de poder, la cual está determinada por la estructura social y, por ende, por las relaciones sociales. Romero (2016) plantea que la base de la violencia de género es la desigualdad y el abuso de poder. Esta comprensión permite al estudiantado comprender la complejidad de la temática en la que está interviniendo, al constituir este un problema social, como también las consecuencias que implica la violencia; no son solo consecuencias individuales, ni afectan solo a quienes se atienden en al Centro, sino es parte de la estructura social. 


\section{Experiencias en violencia intrafamiliar y/o de género}

Los estudiantes reflexionan, a la luz de sus procesos de formación profesional, sobre los episodios de violencia vividos y presenciados en sus familias. A partir de lo cual resignifican las experiencias vividas en pro de contribuir a través de su labor como futuros trabajadores sociales:

mi papá, es bien autoritario, conservador y es violento, él tampoco lo acepta, no lo asimila, en este caso es violento psicológicamente, tiene conceptos autoritarios (...), de autoridad, como la forma no apropiada, y también con su familiares, con las relaciones de sus abuelos, no son violentos, pero uno analizando el concepto de "violencia" en toda su envergadura, sí son violentos, el trato que tienen, es violento, y yo, no sé hace diez años no lo asimilaba, o incluso hace cinco años no asimilaba. Ahora sí. Porque estoy más informado, más capacitado en el tema de violencia. (Ts-5H).

Cada estudiante, mediante su praxis, desnaturaliza comportamientos violentos que anteriormente no problematizaba, logra comprender expresiones, delimita diversos tipos de violencia, no solo la violencia física. El estudiante es capaz de identificar que existen comportamientos sutiles que van en desmedro del otro, reconoce que estos interfieren en la comunicación y en la interacción. Esta comprensión trae consigo un cuestionamiento a la comunicación familiar. Tanto mujeres y hombres en formación profesional resignifican sus historias familiares, a través de esto se "pone en duda versiones del mundo dominantes, imperantes y posiblemente naturalizadas, dogmatizadas" (Molina, 2013).

Se producen procesos de concientización de episodios de violencia al interior de las familias. Se conjugan conocimientos y experiencias, al evocar y reconstruir la propia historia; se identifica el rol de la violencia en sus historias de vida, lo que conlleva un cuestionamiento a la comunicación familiar; se proponen otros modos de vinculación, con el fin de construir relaciones e interacciones sin violencia.

\section{La identificación con los usuarios y usuarias}

Los estudiantes de Trabajo Social, al estar en un estado de constante rememoración y cuestionando la violencia en sus propias historias, reflexionan sobre la dificultad de separar su propia historia de vida y la violencia que observan a través de los procesos de intervención. Son 
capaces de establecer las similitudes entre ambas experiencias: las propias y las de las personas que atienden en sus prácticas profesionales. El dolor de la persona atendida refleja el dolor propio, en palabras de una estudiante:

la otra vez me paso, un usuario decía como "no es que llegaba y hacía esto" y yo me acordaba de lo que le hacía mi papá a mi mamá... entonces, así como no puedo estar pensado en esto (se emociona), entonces eso, como que, igual me ha mantenido como un poco inestable emocionalmente. (Ts-2M)

Según Byung-Chul Han (2012), la inestabilidad provocada por la identificación con la negatividad de los relatos de las personas usuarias, es dada por el exceso de positividad que se constituye en una constante en la sociedad moderna. Convergen las vivencias de las personas usuarias como del estudiantado, ambos experimentan diferentes emociones al concluir las sesiones. A partir de este espacio emerge una emocionalidad asociada al miedo y la rabia, la impotencia al ser testigos a través de la escucha activa de relatos de violencia. La frustración y la impotencia se materializan en los relatos de los jóvenes profesionales en práctica.

Entonces, yo quedaba así como... como con pena, como, como con angustia, cómo, cómo la ayudo, cómo hago esto, y en el fondo empecé a separar un poco las cosas, como que... me limité a lo que se me pedía acá, igual hice derivaciones, tuve que hacer coordinaciones entre redes y todo, pero, pero entendí que en el fondo siempre voy a tener que... esforzarme por separar lo profesional de mi vida personal. (Ts-6M)

Los sentimientos y emociones que se desencadenan durante las intervenciones, producto de esta identificación con las historias de violencia, son también el agotamiento y la desesperanza, muchos de ellos relativos a experiencias no resueltas de violencia.

\section{Estudiantes de Psicología}

En particular para quienes estudian Psicología, la realización de intervenciones en violencia, en el marco de sus prácticas profesionales, implica el despliegue de estrategias para alejar o minimizar la afectación que les produce el rememorar su propia historia. Este modo de enfren- 
tar la intervención y el lugar de la violencia en sus propias experiencias implican cambios más progresivos, reflexivos y pausados.

\section{Conocimientos adquiridos sobre violencia intrafamiliar y de género}

Los conocimientos que se adquieren a través de los contextos de práctica profesional y la adquisición de herramientas en la intervención, permite al estudiantado de Psicología ampliar los repertorios de respuesta frente a la violencia. A partir de estos evidencian transformaciones en las estrategias de interacción de género.

... cambió mi forma de, de entender las relaciones, principalmente las de pareja, yo antes, claro he tenido varias parejas, me ha servido para identificar, en qué situaciones pude haber sido violento, o pudieron haber sido violentas conmigo también, entonces es como, hartas cosas en realidad, como que es realmente ver el mundo desde otra perspectiva, como en ciento ochenta grados. (Ps-4H)

Visualizar o asumir sus vivencias de violencia en sus historias personales, a través de la intervención, posibilita al estudiantado contactarse con sentimientos de vulnerabilidad (Araya y Villena, 2013). Se rememoran sensaciones, sentimientos y emociones, o episodios de sus vidas, que luego del aprendizaje, al alero del ejercicio realizado en sus prácticas profesionales, pueden identificar como violentos.

Asimismo, la compresión de la violencia desde una perspectiva más amplia, producto del ejercicio en dupla, y del aprendizaje de la violencia en sus dimensiones más allá de lo psicológico, es decir, la comprensión de la violencia como un problema estructural, con base en las relaciones e interacciones sociales, permite a los estudiantes despojarse de una visión estereotipada, tanto de quien sufre como de quien ejerce violencia. Es decir, se rompe con la comprensión de la violencia como problemática de nivel individual o psicológica: esta no es una problemática intrapsíquica. Esta comprensión implica un cuestionamiento a la normalización de la violencia en sus propios procesos vitales.

\section{Experiencias en violencia intrafamiliar y/o de género}

La desnaturalización de la violencia de género en el marco de las prácticas profesionales, activa agencias más críticas: se abre una compren- 
sión de la violencia estructural que se perpetúa en las desigualdades de poder (Galtung, 1969). La práctica profesional en violencia también activa, en los estudiantes de Psicología, una revisión e identificación de la violencia en sus propias vidas, en palabras de una estudiante:

es un proceso difícil, si uno lo ve en la familia, lo que es violencia. Mi tía, a veces, llamaba a los carabineros a las doce de la noche para que pudieran ir, y decía que tenía miedo. Y ahora yo lo pienso, y digo, pucha en ese momento, yo no tomaba el peso a al tema. Que me dijera que tiene miedo, así como me lo tomo ahora. Y la verdad es que acá, en el Centro, te pones a pensar en todo eso. (PS- 5M)

La confluencia de la violencia en los relatos propios y de otros, como el cuestionamiento a la violencia en sus diversas formas, en la comprensión de esta como parte de las estructuras de desigualdad de género, que se abre en el marco del desarrollo de la práctica profesional en el Centro de Violencia, activa, sin duda, una comprensión más compleja y próxima del fenómeno. A través de las intersecciones entre la vida de otros y la propia, por medio de la escucha activa, se visibiliza la violencia, se hace evidente la violencia que había sido naturalizada en la propia vida.

\section{La identificación con los usuarios y usuarias}

$\mathrm{Al}$ rememorar y cuestionar la violencia en su propia historia, a partir de la escucha del relato del otro, se produce una identificación con las personas en atención:

mi mamá, que también sufrió violencia, y entonces como que de repente, hay muchas ocasiones... como que... como que la vi a ella, reflejada en esta persona, entonces como que ahi en un momento me tuve que contener y eso fue como lo más significativo, porque era muy similar a lo que había vivido mi mamá. (PS-3M)

A partir de los relatos del estudiantado en práctica de Psicología, se observa que estos despliegan mecanismos de autoprotección, con el fin de alejarse de la temática de violencia. Establecen límites con los usuarios, de modo de protegerse de la identificación con las personas usuarias a partir de los relatos de violencia; se despliegan sentimientos de inseguridad, miedo, desesperanza, preocupación por las consecuencias de la violencia en los usuarias. La sensación de no contar con 
herramientas para manejar su propia identificación genera inseguridad, la sensación de colapso.

Esta inseguridad y sentimientos de indefensión profesional en los practicantes de Psicología están enmarcados en dos hechos que resulta indispensable subrayar: el primero es que el espacio de practica en el Centro de Prevención y Tratamiento de la Violencia Intrafamiliar es para ellos el primer acercamiento al ejercicio profesional durante su proceso de formación; y, como segundo aspecto, este espacio de práctica implica un primer acercamiento a la problemática de la violencia de género y, con ello, la incorporación de otras dimensiones asociadas a esta problemática.

\section{Reflexiones finales}

La producción de agencia, a partir del ejercicio de práctica profesional en violencia de género de los estudiantes de Psicología y Trabajo social, conlleva un impacto en las interacciones y las relaciones al interior de sus familias.

En el caso del estudiantado de Trabajo Social, la agencia se observa en el despliegue de cuestionamientos en la comunicación al interior de sus familias. Al interior de estas se concientiza respecto de episodios de violencia vividos, contribuyendo con ello a generar cambios en sus contextos familiares. Se asume una conducta activa en transformar el uso de la violencia en las interacciones, se pone límite al uso de la violencia en su entorno, se asume una respuesta activa ante situaciones de conflicto y se busca resolverlos sin violencia.

La agencia en los estudiantes de Psicología se pone de manifiesto en el cuestionamiento de las conductas violentas, como en el uso de la violencia para resolver conflictos; sin embargo, se produce una separación entre el mundo de la vida - la familia - y la intervención profesional. Se separa el rol como profesional de la psicología y su papel en el contexto familiar y con sus pares.

Para ambos practicantes, tanto desde el Trabajo Social como desde la Psicología, se producen procesos de identificación con las personas con quienes se interviene. Asimismo, esta identificación se refleja en los procesos de cambio que el estudiantado activa al interior de sus familias, de modo de intentar modificar sus dinámicas familiares. 
Este proceso de introspección que conlleva una emocionalidad asociada, para Byung-Chul Han (2012) se relaciona con la identificación con los y las usuarias, ya que "no solamente veo lo otro, sino que también lo soy, y «lo otro es al mismo tiempo yo»"(p. 47), produciendo en los participantes "un cansancio en cuanto volverse accesible, es más, como consumación del hecho de ser tocado y poder tocar a su vez" (Ibid.). Cuestionar el rol de la violencia en su historia familiar e intervenir en violencia de género es una forma de sobreexplotación, la que, desde el autor, se entiende como el "exceso de estímulo, informaciones e impulsos" (p. 21), al exponerse constantemente a personas que presentan relatos de violencia, relatos de tristeza, angustia, dolor, extenuación, miedo, etc.

En estos procesos se genera también un estado de "flexplotación", planteado por Bourdieu (1999), que se entiende como un estado de constante inseguridad, que obliga a los trabajadores a la sumisión y a la tolerancia de la explotación. Estado que experimentan los estudiantes al intervenir en violencia, como parte del proceso formativo-práctico. A esto se suma la producción de agencias que les sitúan diferenciadamente en sus entornos en relación con la violencia de género. Este es un periodo de alto cuestionamiento y reflexión en torno a las desigualdades estructurales y el lugar que ellos y ellas ocupan en estas.

Se hace patente la necesidad de continuar investigando acerca de la construcción de agencias en estudiantes en prácticas profesionales que sean padres o madres. Es posible suponer que en ellos la construcción de esta agencia, asociada al ejercicio de formación profesional, estará relacionada con el cuestionamiento en los procesos de crianza de sus hijos. Creemos que se requiere profundizar en esta comprensión, pues la evidencia que hemos recogido da cuenta del impacto del estudiantado en sus familias de origen, y no hemos abordado el impacto en la construcción de nuevas familias y en los roles que pudieran asumir como progenitores en los procesos de socialización de género.

Debemos subrayar lo indispensable de asegurar espacios de autocuidado y de reflexión colectiva, a través de los cuales los estudiantes sean parte de procesos de introspección profunda, que les permitan reelaborar vivencias en relación con la violencia de género y construir un marco emocional que sostenga intervenciones de alta complejidad. Los relatos evidencian expresiones de Síndrome de Burnout, Estrés 
Traumático Secundario y Estados de Extenuación Emocional. Estas condiciones de estrés, por lo general, son descritas por profesionales egresados y que trabajan estos temas; sin embargo, el estudio muestra que se producen también en practicantes en procesos de formación profesional, pues es la identificación con los relatos de los usuarios lo que las desencadena y no el tiempo de exposición.

Es relevante señalar la importancia de este tipo de estudio para las disciplinas de Trabajo Social y Psicología. Debemos revisar los mecanismos de formación, el impacto de estos procesos de formación a través de la intervención, las complejidades que implica el despliegue de habilidades y estrategias de alta complejidad disciplinar, y el desafío de una intervención psicosocial. Aún sigue siendo un desafío la despalotogización de la violencia de género y la comprensión de ésta en marcos de relaciones de desigualdad estructural, en los que el Trabajo Social debe abordar estas problemáticas desde perspectivas feministas críticas.

Cabe destacar finalmente el valor de estos espacios de formación institucional, en tanto el ejercicio práctico interdisciplinar contribuye a la emergencia de agencias en los estudiantes, trascendiendo no solo en lo formativo, sino que en la manera en que estos hombres y mujeres se posicionan de forma activa, crítica y reflexiva en la estructura social frente a la violencia de género.

\section{Bibliografía}

Araya, P. y Villena, S. (2013). El cuidado del trauma: Una aproximación a las experiencias de trabajo de terapeutas especializados en atención a víctimas de violencia sexual. (Tesis para optar al título de Psicología). Universidad de Chile.

Arita, B., Castillas, Q. y Romero, R. (s.f.). Estrés traumático secundario y salud en la intervención relacional de trabajadores sociales. Recuperado de: https://eventos.ucol.mx/content/micrositios/241/file/memoria/pdf/30.pdf

Bivort, B. (2005). Estrategias de superación de la pobreza: Agencia, ciudadanía y redes en el programa puente. Theoria, 14(2), 9-16. Recuperado de: http://www.ubiobio.cl/theoria/v/ v15/al.pdf

Bonilla, M. y López, A. (2016). Ejemplificación del proceso metodológico de la teoría fundamentada. Cinta de Moebio, (57), 
305-315. Recuperado de: https://scielo.conicyt.cl/scielo. php?script=sci_arttext\&pid=S0717554X2016000300006

Bourdieu, P. (1980). Sentido práctico. Buenos Aires: Siglo XXI Editores.

Bourdieu, P. (1994). Razones prácticas sobre la Teoría de la Acción. París: Editorial Anagrama.

Bourdieu, P. (1999). Contrafuego: Reflexiones para servir a la resistencia contra la invasión neoliberal. Barcelona: Editorial Anagrama.

Burgos, D. (2013). Efectos de la problemática presentada por el paciente en la vida personal de psicólogos novatos y experimentados y sus mecanismos para elaborar los procesos y/o cuestionamiento elicitados. (Tesis pregrado para optar al título de psicólogo). Universidad de Chile.

Canevaro, A., Abeliuk, Y., Condeza, M., Escala, M., García, G. y Montes, J. (2016). El nacimiento del terapeuta sistémico. El trabajo directo con las familias de origen de los terapeutas en formación. Revista de psicoterapia relacional e intervenciones sociales, (31), 127-145.

Cañas, A. (2007). Caracterización teórico-metodológica de las intervenciones grupales con mujeres víctimas de violencia en la pareja. (Tesis para optar al título de Psicóloga). Universidad de Chile.

Cárdenas, J. (2018). Una reflexión de la vivencia de un maestro, desde el concepto de resignificación, a partir de la apropiación del microrrelato como técnica de investigación. Aletheia, 10(1), 44-57.

Carvajal, C. (2012). Desarrollo de la persona del terapeuta y su influencia en el quehacer profesional: Desde la mirada de los experimentados. (Tesis para optar al grado de Magíster en Psicología clínica). Universidad de Chile.

Castillas, O. (2016). Estrés traumático secundario y salud en la atención profesional con familias: Una mirada relacional. México: Santiago, 1(139), 95-109.

Claramunt, C. (1999). Ayudándonos para ayudar a otros: Guía para el autocuidado de quienes trabajan en el campo de la violencia intrafamiliar. Serie género y salud pública. San José, Costa Rica: Organización Panamericana de la Salud, Programa mujer, salud y desarrollo. 
Cuñat, R. (2007). Aplicación de la teoría fundamentada (grounded theory) al estudio del proceso de creación de empresas. Recuperado de: https://dialnet.unirioja.es/servlet/ articulo? codigo $=2499458$

Daskal, A. (2017). La persona del terapeuta. Chile: Ediciones UC.

Filgueira, M. (2014). Síndromes profesionales del psicoterapeuta: Propuestas de autocuidado con psicodrama. Psicoterapia y Psicodrama, 3(1), 37-78.

Fowler, W. R. (2013). El pensamiento de Pierre Bourdieu: Apuntes para una mirada arqueológica. Revista de Museología Kóot, 117-135.

Galtung, J. (1969). Violence, Peace, and Peace Research. Journal of Peace Research.

Han, B-Ch. (2012). La Sociedad del Cansancio. Argentina: Herder.

Hernández, R., Fernández, C. y Baptista, M. (2010). Metodología de la Investigación. México D.F: McGraw-Hill.

Jiménez, J., Pallares, D. y Bustamante, M. (2005). Interdisciplinariedad y formación en Trabajo Social. Revista Tendencias \& Retos, (10), 115-127. Recuperado de: https://dialnet.unirioja.es/ ejemplar/385000

Lagarde, M. (1996). Cuadernos inacabados, género y feminismo: Desarrollo humano y democracia. Madrid: Horas y Horas.

Lamas, M. (2000). Diferencias de sexo, género y diferencia sexual. Cuicuilco, 7(18).

Maslach, C. \& Jackson, S. (1981). Maslach Burnout Inventory (2a ed.). Palo Alto, CA.: Consulting Psychologist Press.

Moix, M. (2004). El trabajo y los servicios sociales: su concepto. Cuadernos de trabajo social, (17), 131-141.

Molina, N. (2013). discusiones acerca de la resignificación y conceptos asociados. Revista MEC-EDUPAZ, Universidad Nacional Autónoma de México, (3). Recuperado de: http:// www.journals.unam.mx/index.php/mecedupaz/article/ view/36436/33013

Peláez, M., Cañon, O. y Noreña, N. (2007). La intervención psicosocial en un contexto investigativo: "Lecturas psico-sociales sobre jóvenes agrópolis - sector rural- desde diversos actores que los intervienen". Aletheia, (25), 191-202. Recuperado de: http://pepsic.bvsalud.org/scielo.php?script=sci_arttext\&pi $\mathrm{d}=$ S141303942007000100015\&lng=pt\&tlng=es . 
Romero, S. (2016). La violencia contra la mujer en el contexto de pareja, visto desde el Trabajo Social Chileno. Santiago de Chile: Ediciones Universidad Tecnológica Metropolitana.

Ruiz, J. (2012). Metodología de la investigación cualitativa. Bilbao: Universidad de Deusto.

SERNAMEG. (s.f.). Misión y funciones. Recuperado de: http://www.sernameg.gob.cl/?page_id=25

Szmulewicz, T. (2013). La persona del terapeuta: eje fundamental de todo proceso terapéutico. Revista chilena neuro-psiquiatría, 51(1), 61-69. Recuperado de: https://scielo.conicyt.cl/pdf/ rchnp/v5ln1/art08.pdf

Torre, G., Carlo, E., Florido, A., Opazo, H., Ramírez, C., Rodríguez, P. y Tirado, J. (2011). Teoría Fundamentada o Grounded Theory. Madrid, España: Universidad Autónoma de Madrid.

Vexina, G. (2014). El psicólogo frente a la problemática de la violencia familiar. Los invisibles de la práctica. En XV Congreso argentino de psicología: los atravesamientos en la salud mental, intervenciones de la psicología en los contextos actuales (pp. 54- 59). Argentina: El Calafate.

Zavala, V. (2011). La escritura académica y la agencia de los sujetos. Cuadernos Comillas, 52-66.

Zurh, O. (1994). Psychotherapists and their families: The effect of clinical practice on individual and family dynamics and how to prevent therapists' Burnout and impairment. Recuperado de: https://www.zurinstitute.com/therapists-families/ 